\title{
Three-dimensional analysis of facial asymmetry after zygomaticomaxillary complex fracture reduction: a retrospective analysis of 101 East Asian patients
}

Jakwang Cho,

Youngjun Kim, Youngwoong Choi

Department of Plastic and Reconstructive Surgery, Sanggye Paik Hospital, Inje University College of Medicine, Seoul, Korea
Background: The zygomaticomaxillary complex (ZMC) has a protruded, convex shape and plays a vital role in determining the contour by affecting the width of the middle face. This study aimed to evaluate the efficiency of ZMC fracture reduction and explore detailed directions for outcome improvement.

Methods: We conducted a retrospective study of patients diagnosed with unilateral ZMC fracture who underwent ZMC reduction surgery at a single hospital between January 2015 and May 2020. The primary outcome variable was facial asymmetry using the difference in the bilateral malar eminence (ME) position measured by computed tomography scan. The 3-dimensional distance $\left(\|_{A}\right.$, asymmetry index) and the distance in each dimension, $D_{x}$ (anteroposterior distance), $D_{y}$ (mediolateral distance), and $\mathrm{D}_{2}$ (superoinferior distance) were compared.

Results: A total of 101 patients with ZMC fractures and 54 non-fracture patients were enrolled in the study. The mean age of the study sample was 43.49 years (control sample, 43.35 years), and the male-to-female ratio was 66.3:33.7 (control sample, 64.8:35.2). There were 53 and 48 patients with right and left ZMC fractures, respectively. The $I_{A}$ was not statistically different between the two groups. In terms of position in each dimension, only $D_{x}$ was significantly different between the two groups.

Conclusion: The results show that overall facial asymmetry was recovered after ZMC reduction, but in certain dimension significant difference in ME position has still remained. For further improvement, treatment should be performed to relieve malar depression in the anteroposterior dimension.

Abbreviations: $\mathrm{CT}$, computed tomography; $\mathrm{D}_{\mathrm{x}}$, anteroposterior distance; $\mathrm{D}_{\mathrm{y}}$, mediolateral distance; $\mathrm{D}_{z}$, superoinferior distance; $\mathrm{I}_{\mathrm{A}}$, asymmetry index; $\mathrm{ME}$, malar eminence; ZMC, zygomaticomaxillary complex.

Keywords: Facial asymmetry / Malar bone / Maxillary fracture / Maxillofacial surgery / Zygomatic fracture

\section{INTRODUCTION}

Fractures of the zygomaticomaxillary complex (ZMC) account

\section{Correspondence: Youngwoong Choi}

Department of Plastic and Reconstructive Surgery, Sanggye Paik Hospital, Inje

University College of Medicine, 1342 Dongil-ro, Nowon-gu, Seoul 01757, Korea

E-mail: pshero@gmail.com

Received June 8, 2021 / Revised June 18, 2021 / Accepted June 20, 2021 for approximately $25 \%$ of all facial bone fractures, the second most common after nasal bone fractures, and their surgical treatment has been described extensively [1-3]. In addition, various postoperative complications, including functional (visual impairment, diplopia, malocclusion, and hypoesthesia involving the infraorbital nerve) and esthetic deformities (malar asymmetry, midfacial widening, and enophthalmos) have been 
introduced for decades by a few studies, and efforts to reduce these have been continued [1-5].

ZMC has a protruded and convex shape. Its projection plays an essential role in determining the contour by affecting the width of the middle face [6-8]. It determines the height, width, and projection of the lateral face and shows racial characteristics. East Asian populations, especially Chinese, Korean, and Japanese, are characterized by high and broad cheekbones. Their overly protruding zygomas and zygomatic arches upset the harmony and make their facial contours appear rough, aggressive, and masculine [6-10]. In this context, ZMC reduction poses a continuing challenge for maxillofacial surgeons because of its significant position in facial esthetics and symmetry, requiring skills and expertise. Numerous studies have demonstrated quantitative and qualitative evaluations of the adequacy of facial symmetry recovery after ZMC fracture reduction surgery [2,11-13]. However, there are few quantitative studies on postoperative symmetry recovery after ZMC reduction in East Asian populations with characteristic zygoma shapes.

In this study, we performed a 3-dimensional analysis of the degree of recovery of malar asymmetry after ZMC reduction surgery in patients with unilateral ZMC fracture compared to patients without any facial bone fracture. The specific aims of our study were to (1) evaluate the adequacy of ZMC fracture reduction through quantitative analysis of postoperative computed tomography (CT) scans and (2) explore detailed directions for outcome improvement.

\section{METHODS}

\section{Patient selection}

We conducted a retrospective controlled cross-sectional study of 101 patients diagnosed with unilateral ZMC fracture due to trauma. We underwent ZMC reduction surgery in the Department of Plastic and Reconstructive Surgery at a Sanggye Paik Hospital in Seoul, Korea, by five surgeons between January 2015 and May 2020. This study was approved by the Institutional Review Board (IRB No. 2021-05-013). Written consent was obtained either directly from the patients or their legal representatives.

The inclusion criteria for the study group were: (1) patients who had unilateral ZMC fracture; (2) were fully dentate adults to minimize error caused by unilateral bone resorption and maxillary retrusion of the aging process [14]; (3) had undergone surgical treatment of the ZMC fracture at our institution within 2 weeks of fracture; or (4) had agreed to be enrolled in the study and underwent postoperative CT scan on the day of surgery or the day after. Patients were excluded from the study if they had congenital facial deformities, had a history of facial fractures or facial surgery, had low deviated ZMC fractures, had ZMC fractures with other facial fractures (nasal bone fractures, mandibular fractures, supraorbital rim fractures, etc.).

The control group was composed of patients referred to our institution with superficial facial lacerations, who underwent primary repair, and underwent a CT scan to evaluate the facial bone fractures. The inclusion criteria for the control group were patients who agreed to be enrolled in the study and were matched to the study sample for age and sex ratio. Cases with apparent facial asymmetry, including congenital anomalies, previous facial fracture history, or surgical history, were excluded.

\section{Surgical technique}

All surgeries were performed under general anesthesia via oropharyngeal intubation. A sublabial incision ranging from 4 to 5 $\mathrm{cm}$ was made above the gingivobuccal sulcus, from the lateral incisor to the first molar. Dissection along the subperiosteal plane to visualize the maxilla and expose the infraorbital nerve was performed $[1,15]$. An eyelid incision via the transcutaneous subciliary approach was also made to visualize fractures of the inferior orbital rim. Bevans and Moe [16] described the details of the orbital fracture surgery. After careful reduction of the body of maxilla followed by orbital floor alignment reduction, in most cases, fixation with a curved-shaped microplate with screws (Micro titanium plate 1.2, Micro screw 1.2 [4 mm], Leforte; Jeil Medical Corporation, Seoul, Korea) on the orbital rim and L-shaped microplate and screws (Midface titanium plate 1.6, Midface screw 1.6 [6 mm], Leforte; Jeil Medical Corporation) on the zygomaticomaxillary buttress were performed for optimal ZMC fracture stability (Fig. 1) [1].

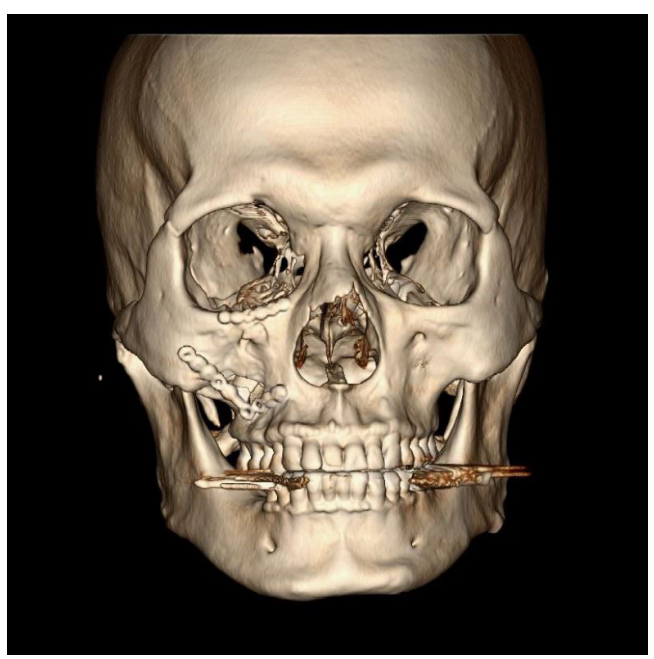

Fig. 1. Frontal view of postoperative 3-dimensional computed tomography image demonstrating restoration of malar asymmetry. 


\section{Variables and data analysis}

For the primary variable, we set the facial asymmetry measured by the difference in the position of malar eminence (ME). As in previous studies [2,17], we defined the ME position as the point of the zygomatic complex protruding from the final cephalo-facial midsagittal plane on CT scan (with the axial cut demonstrating most of the bilateral zygomatic complex region) (Fig. 2).

Before the measurements, 3-dimension reconstruction images after positional adjustment were acquired with commercial software (Aquarius iNtuition, version 4.4.7; Terarecon, Inc.,

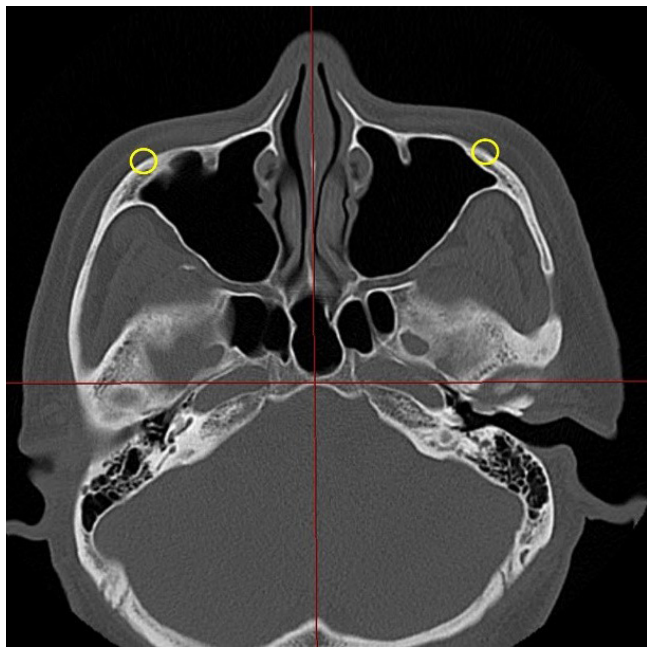

Fig. 2. The axial cut covering most of the bilateral zygomatic complex region. The position of the malar eminence (yellow circles) was defined as the most protruding point of the zygomatic bone on the zygomatic complex from the axial cut covering most of the bilateral zygomatic complex region.

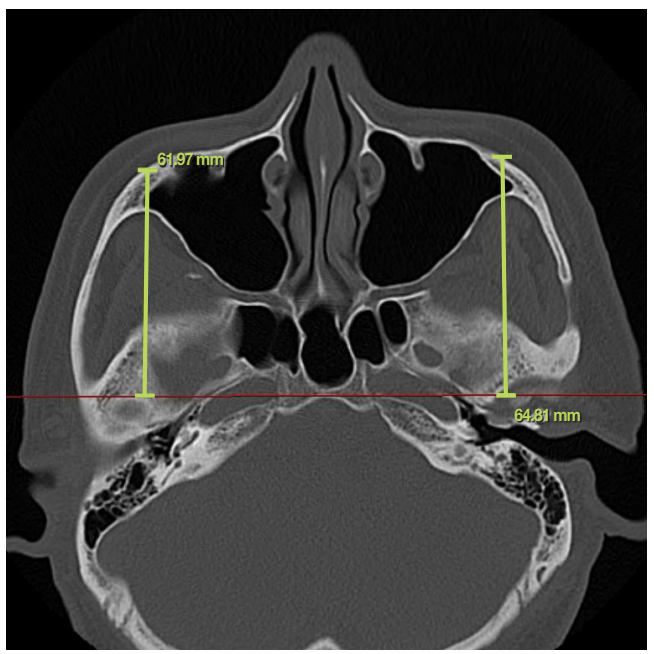

Fig. 3. Symmetry evaluation of the malar eminence using an axial computed tomography view. The difference of bilateral distances from the malar eminence to the coronal plane passing through the anterior edge of the foramen magnum was defined as the anteroposterior distance, $\mathrm{D}_{\mathrm{x}}$.
Foster City, CA, USA) in order to reduce the error according to head position during CT scan. Using the software, each plane was determined based on anatomical landmarks. The midsagittal plane was first determined by nasion, sella and gnathion. The horizontal plane was then generated perpendicular to the sagittal plane and rotated until it was parallel to the Frankfort horizontal plane. The coronary plane was set perpendicular to the two existing planes [13].

We compared the 3-dimensional ME positions on both sides of the control group and the study group using postoperative

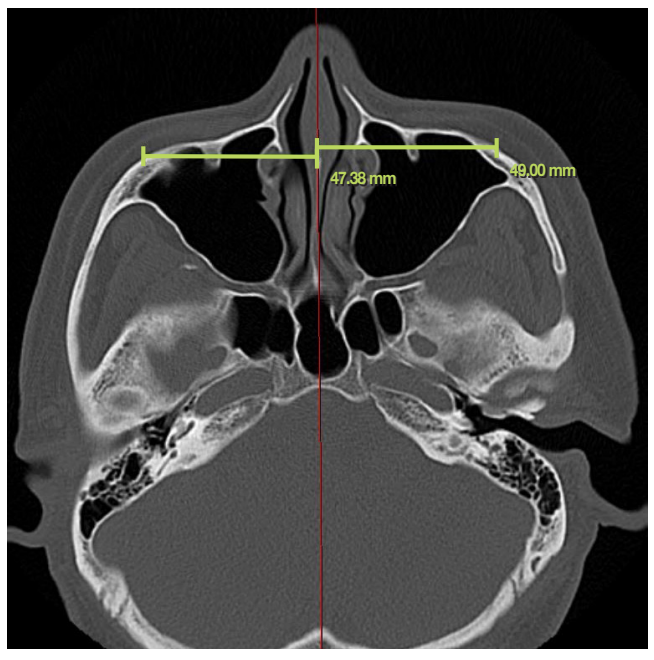

Fig. 4. Symmetry evaluation of the malar eminence using an axial computed tomography view. The difference of bilateral distances from the malar eminence to the midsagittal plane was defined as the mediolateral distance, $D_{y}$.

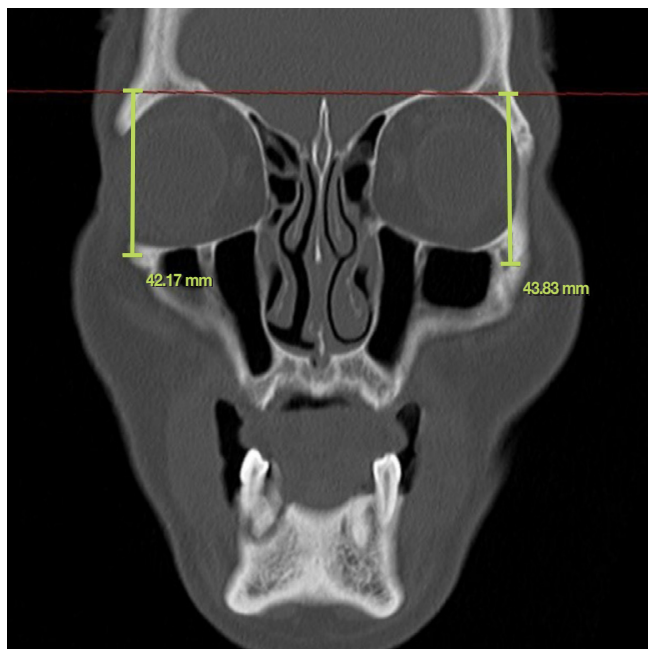

Fig. 5. Symmetry evaluation of the malar eminence using a coronal computed tomography view. The difference of bilateral distances from the malar eminence to a transverse plane passing through bilateral superior orbital rims was defined as the mediolateral distance, $\mathrm{D}_{z}$ 
CT scans. The difference in the linear distances from the bilateral ME position to the reference planes was measured and marked according to the reference planes as follows: a coronal plane passing through the anterior edge of the foramen magnum (anteroposterior distance, $\mathrm{D}_{\mathrm{x}}$ ) (Fig. 3), the midsagittal plane (mediolateral distance, $D_{y}$ ) (Fig. 4), and the transverse plane passing through bilateral superior orbital rims (superoinferior distance, $D_{z}$ ) (Fig. 5). $D_{x}$ and $D_{y}$ were measured at the axial cut of the CT scan. In the case of $\mathrm{D}_{\mathrm{z}}$, the ME point was converted from axial to coronal cut using a 3-dimensional cursor in the picture archiving and communications system viewer program (Marosis m-view 5.4; Marotech, Seoul, Korea), and the distance was measured. Finally, to compare the 3-dimensional position difference between bilateral ME positions, the asymmetry index $\left(\mathrm{I}_{\mathrm{A}}\right)$ was measured according to the following formula as described by Ras et al. [18]

$$
\mathrm{I}_{\mathrm{A}}=\sqrt{\left(\mathrm{D}_{\mathrm{x}}^{2}+\mathrm{D}_{\mathrm{y} 2}+\mathrm{D}_{\mathrm{z}}^{2}\right)}
$$

The other variables of this study were demographic and anatomic variables. These variables included age (years) and sex (male or female) for explanatory purposes and were matched between the two groups. Anatomical variables included the fracture side (left or right).

All measurements were performed twice by one investigator at least 24 hours apart for internal validation. Data were analyzed using SPSS 25.0 (IBM Corp., Armonk, NY, USA) with the significance level set at $p<0.05$. The mean values of the variables were compared using an independent $t$-test between the two groups.

\section{RESULTS}

During the study period, 101 patients with ZMC fractures and 54 non-fracture patients were enrolled. The mean age of the study sample was 43.49 years (range, $17-80$ years), and the male-to-female ratio was 66.3:33.7. There were 53 and 48 patients with right and left ZMC fractures, respectively. The mean age of the control sample was 43.35 years (range, 18-79 years), and the male-to-female ratio was 64.8:35.2, which was matched with the study group. In addition, the age distribution was also matched between the two groups (Table 1).

Among the study group patients, six patients (5.94\%) received microplate fixation only on the zygomaticomaxillary buttress, six patients (5.94\%) received microplate fixation only on the orbital rim, and 89 patients $(88.12 \%)$ received 2-point fixation by fixing both sites. Before receiving surgical treatment, the $\mathrm{I}_{\mathrm{A}}$ and all other variables $\left(D_{x}, D_{y}\right.$, and $\left.D_{z}\right)$ of the study group showed statistically significant differences compared to the control
Table 1. Demographic features of study population

\begin{tabular}{lcc}
\hline Variable & Control group $(n=54)$ & Study group $(n=101)$ \\
\hline Age (yr) & $43.35 \pm 16.70(18-79)$ & $43.49 \pm 17.35(17-80)$ \\
Age group (yr) & $17(31.5)$ & $32(31.7)$ \\
$<30$ & $6(11.1)$ & $13(12.9)$ \\
$30-39$ & $11(20.4)$ & $18(17.8)$ \\
$40-49$ & $10(18.5)$ & $17(16.8)$ \\
$50-59$ & $10(18.5)$ & $21(20.8)$ \\
$\geq 60$ & & \\
Sex & $35(64.8)$ & $67(66.3)$ \\
Male & $19(35.2)$ & $34(33.7)$ \\
Female & & $53(52.5)$ \\
ZMC fracture side & - & $48(47.5)$ \\
Right & - & \\
Left & & \\
\hline
\end{tabular}

Values are presented as mean $\pm \mathrm{SD}$ (range) or number (\%). ZMC, zygomaticomaxillary complex.

Table 2. Bilateral malar eminence position differences in study and control patients

\begin{tabular}{llll}
\hline $\begin{array}{l}\text { Measurements } \\
(\mathrm{mm})\end{array}$ & \multicolumn{1}{c}{ Study group $(\mathrm{n}=54)$} & Control group $(\mathrm{n}=101)$ & $p$-value \\
\hline $\mathrm{D}_{x}$ & $1.84 \pm 1.49(0-6.90)$ & $1.36 \pm 1.08(0.02-4.00)$ & $<0.03$ \\
$\mathrm{D}_{y}$ & $2.46 \pm 1.89(0-9.37)$ & $2.21 \pm 1.65(0.06-5.67)$ & $>0.05$ \\
$\mathrm{D}_{z}$ & $2.47 \pm 2.13(0-8.90)$ & $2.55 \pm 1.82(0.03-7.86)$ & $>0.05$ \\
$\mathrm{AA}^{\mathrm{a}}$ & $4.56 \pm 2.26(0.78-12.34)$ & $4.10 \pm 1.91(0.70-9.44)$ & $>0.05$ \\
\hline
\end{tabular}

Values are presented as mean $\pm \mathrm{SD}$ (range).

$\mathrm{D}_{\mathrm{x}}$, anteroposterior distance; $\mathrm{D}_{\mathrm{y}}$, mediolateral distance; $\mathrm{D}_{\mathrm{z}}$, superoinferior distance.

a) Asymmetry index $\left(l_{A}\right)=\sqrt{D_{x}^{2}+D_{y}{ }^{2}+D_{z}^{2}}$

group $(p<0.05)$, suggesting that asymmetry of the ME position was evident in patients with ZMC fracture.

The mean and standard deviations of the bilateral ME position differences in each dimension of the control and study groups after reduction surgery are indicated in Table 2 . The results showed that there was malar asymmetry also in the control group in some extent. The mean $\mathrm{I}_{\mathrm{A}}$ in control group was $4.10 \pm 1.91 \mathrm{~mm}$ indicating that the ME position can show a difference normally as much as the corresponding value. The $\mathrm{I}_{\mathrm{A}}$ in the study group $(4.56 \pm 2.26 \mathrm{~mm})$ was not statistically different from that of the control group $(p>0.05)$. In terms of position in each dimension, both $\mathrm{D}_{\mathrm{y}}$ and $\mathrm{D}_{\mathrm{z}}$ did not differ significantly ( $p>0.05$ ), but $\mathrm{D}_{\mathrm{x}}$ was significantly different between the two groups $(p<0.03)$.

\section{DISCUSSION}

In this study, we evaluated whether cosmetic surgery was appropriately performed by assessing ME symmetry after open reduction of ZMC fractures compared to those of healthy patients 
without fractures. We hypothesized that ME symmetry would be restored by reduction surgery for ZMC fractures. In addition, we tried to find detailed shortcomings through quantitative analysis of each dimension with a CT scan. We also attempted to find a method to improve the surgical results by this review.

There is no consensus on the ideal reference axis and plane using CT scans in the craniofacial system. Still, Rudderman and Mullen [19] suggested that there should be no translations or rotations of the $\mathrm{x}, \mathrm{y}$, and $\mathrm{z}$ axes to describe the location of the fracture and the deviation of the bone [13]. Otherwise, due to undetected rotation of the axis, the ZMC displacement may be ignored or inadequately evaluated. In this study, we used threedimension reconstruction images for positional adjustment with known landmarks (nasion, sella, gnathion and Frankfort horizontal plane) [13]. Three or more landmarks (Anterior border of foramen magnum, superior orbital rims and cephalofacial midsagittal plane) were used to define the relative planes and axes. Results showed the adequacy of ZMC fracture reduction and present detailed suggestion for outcome improvement. After ZMC reduction surgery, the symmetry of the ME position was significantly restored, but there were some bilateral differences. Still, these differences were found to be similar to those found in the control group of patients without fracture. Patients without fracture can also show a different position of ME of about $4.10 \mathrm{~mm}$ on average. Patients' awareness of this fact can reduce their dissatisfaction.

Pau et al. [17] reported that normal subjects showed more malar position asymmetry in the anteroposterior dimension $(1.83 \pm 1.55 \mathrm{~mm})$ than in mediolateral dimension and in superoinferior dimension through a study of patients admitted to a university hospital in California, USA. However, in our study, which included 54 East Asian (Korean) normal subjects without fracture, the anterior to posterior dimension showed less asymmetry $(1.36 \pm 1.08 \mathrm{~mm})$ than in other two dimensions. The difference in distance was smaller in $\mathrm{D}_{\mathrm{x}}$ than in $\mathrm{D}_{\mathrm{y}}$ and $\mathrm{D}_{z}$, which can be attributed to differences in the shape of cheekbones according to race [6-8].

In the detailed description of malar asymmetry including soft tissue reported by Kim et al. [12], a statistically significant malar depression was observed even after surgery in anteroposterior dimension. Kim et al. [12] reported no significant difference in bilateral position of hard tissue landmarks after open reduction and internal fixation in patients with ZMC fracture, but soft tissue depression was confirmed after surgery. Soft tissue depression around zygoma would be more noticeable if reduction were not accurate and was more noticeable when titanium was used in comparison to biodegradable plates [12]. Considering the results of our study and the occurrence of soft tissue depression after surgery, we carefully suggest an overcorrection surgical technique and follow-up studies about the methods of overcorrection technique are needed.

Comparison of the outcomes of 2-point (at infraorbital margin and zygomaticofrontal buttress region) versus 3-point internal fixation (at frontozygomatic suture, infraorbital margin, and zygomaticomaxillary buttress region) in zygomaticomaxillary reduction remains controversial. We did not perform more than two fixations for reduction surgery. Nasr et al. [20] reported no significant difference between 2-point fixation and 3-point fixation for ME asymmetry, including zygomatic projection and width. In radiological assessment using CT scan 3 months after surgery to evaluate post-reduction displacement, backward displaced zygoma reduction in axial view was significantly better in the 2-point fixation group than in the 3-point fixation group. In addition, the total cost of the plates and screws used was significantly higher with 3-point repair than with 2-point repair. However, several studies have demonstrated that the 3-point rigid fixation of the fractured ZMC provides better stability against masticatory forces and maintains anatomic position with less linear and rotational translation [21,22].

This study had some limitations. It can be pointed out that the study did not include soft tissue, which may be of importance in determining aesthetic facial symmetry and appearance. In addition, the fact that the subjective evaluation and satisfaction of the outcome were not investigated is also a limitation. Gaziri et al. [11] evaluated hard tissue asymmetry in ZMC fracture patients and reported that patients often complained about their soft tissue asymmetry even though the bone fragments were accurately reduced. In postoperative malar asymmetry, the patient's subjective satisfaction is essential because it is an aesthetic sequela rather than a functional sequela. In this study, we evaluated the adequacy of restoration of the symmetry of hard tissue. We also conducted a study on the restoration of soft tissue symmetry according to the patient's subjective satisfaction and sought ways to improve the outcome.

\section{NOTES}

\section{Conflict of interest}

No potential conflict of interest relevant to this article was reported.

\section{Ethical approval}

The study was approved by the Institutional Review Board of Inje University Sanggye Paik Hospital (IRB No. 2021-05-013) and performed in accordance with the principles of the Declaration of Helsinki. Written informed consent was obtained. 


\section{Patient consent}

The patients provided written informed consent for the publication and the use of their images.

\section{ORCID}

Jakwang Cho

https://orcid.org/0000-0003-4707-2901

Youngjun Kim

https://orcid.org/0000-0001-7797-248X

Youngwoong Choi

https://orcid.org/0000-0002-2211-2981

\section{Author contribution}

Conceptualization: YC. Data curation: YK, YC. Formal analysis: JC. Visualization: JC. Writing - original draft: JC. Writing review \& editing: YK.

\section{REFERENCES}

1. Strong EB, Gary C. Management of zygomaticomaxillary complex fractures. Facial Plast Surg Clin North Am 2017;25:54762.

2. Khaqani MS, Tavosi F, Gholami M, Eftekharian HR, Khojastepour L. Analysis of facial symmetry after zygomatic bone fracture management. J Oral Maxillofac Surg 2018;76:595-604.

3. Yoon T, Choi Y, Cho J, Kim Y, Nam S. Primary infraorbital foramen decompression for the zygomaticomaxillary complex fracture: is it essential? J Craniofac Surg 2016;27:61-3.

4. Hong WT, Choi JH, Kim JH, Kim YH, Yang CE, Kim J, et al. Trigeminal somatosensory evoked potential test as an evaluation tool for infraorbital nerve damage. Arch Craniofac Surg 2019;20:223-7.

5. van den Bergh B, Karagozoglu KH, Heymans MW, Forouzanfar T. Aetiology and incidence of maxillofacial trauma in Amsterdam: a retrospective analysis of 579 patients. J Craniomaxillofac Surg 2012;40:e165-9.

6. Chen T, Hsu Y, Li J, Hu J, Khadka A, Wang Q, et al. Correction of zygoma and zygomatic arch protrusion in East Asian individuals. Oral Surg Oral Med Oral Pathol Oral Radiol Endod 2011;112:307-14

7. Zou C, Niu F, Liu J, Chen Y, Wang M, Su R, et al. Midface contour change after reduction malarplasty with a modified Lshaped osteotomy: a surgical outcomes study. Aesthetic Plast Surg 2014;38:177-83.

8. Jirawatnotai S, Sriswadpong P. The difference in the location of the malar summit between genders in Southeast Asians with appropriate references. Arch Craniofac Surg 2021;22:78-84.

9. Onizuka T, Watanabe K, Takasu K, Keyama A. Reduction ma- lar plasty. Aesthetic Plast Surg 1983;7:121-5.

10. Lee YJ, Lee IS, Yang HJ. Reduction malarplasty combined with facelift via the prezygomatic space. Arch Aesthetic Plast Surg 2020;26:138-43.

11. Gaziri DA, Omizollo G, Luchi GH, de Oliveira MG, Heitz C. Assessment for treatment of tripod fractures of the zygoma with microcompressive screws. J Oral Maxillofac Surg 2012; 70:e378-88.

12. Kim DH, Kim RH, Lee J, Chee YD, Kwon KH. Evaluation of soft tissue asymmetry using cone-beam computed tomography after open reduction and internal fixation of zygomaticomaxillary complex fracture. J Korean Assoc Oral Maxillofac Surg 2014;40:103-10.

13. Mao SH, Hsieh YH, Chou PY, Shyu VB, Chen CT, Chen CH. Quantitative determination of zygomaticomaxillary complex position based on computed tomographic imaging. Ann Plast Surg 2016;76 Suppl 1:S117-20.

14. Mendelson $\mathrm{B}$, Wong $\mathrm{CH}$. Changes in the facial skeleton with aging: implications and clinical applications in facial rejuvenation. Aesthetic Plast Surg 2012;36:753-60.

15. Ji SY, Kim SS, Kim MH, Yang WS. Surgical methods of zygomaticomaxillary complex fracture. Arch Craniofac Surg 2016; 17:206-10

16. Bevans SE, Moe KS. Advances in the reconstruction of orbital fractures. Facial Plast Surg Clin North Am 2017;25:513-35.

17. Pau CY, Barrera JE, Kwon J, Most SP. Three-dimensional analysis of zygomatic-maxillary complex fracture patterns. Craniomaxillofac Trauma Reconstr 2010;3:167-76.

18. Ras F, Habets LL, van Ginkel FC, Prahl-Andersen B. Method for quantifying facial asymmetry in three dimensions using stereophotogrammetry. Angle Orthod 1995;65:233-9.

19. Rudderman RH, Mullen RL. Biomechanics of the facial skeleton. Clin Plast Surg 1992;19:11-29.

20. Nasr WF, ElSheikh E, El-Anwar MW, Sweed AH, Bessar A, Ezzeldin N. Two-versus three-point internal fixation of displaced zygomaticomaxillary complex fractures. Craniomaxillofac Trauma Reconstr 2018;11:256-64.

21. Mahmood HN, Rahim AU, Khan WU. Outcome of treatment of zygomatic bone fracture by two point fixation versus three point fixation in Mayo Hospital Lahore. J Pak Med Assoc 2019; 69:1623-7.

22. Degala S, Radhakrishna S, Dharmarajan S. Zygomaticomaxillary fracture fixation: a prospective comparative evaluation of two-point versus three-point fixation. Oral Maxillofac Surg 2021;25:41-8. 